Research Article

\title{
Fault Dynamic Modeling and Characteristic Parameter Simulation of Rolling Bearing with Inner Ring Local Defects
}

\author{
Zhang Fengling, Zhang Yuwei, Guan Jiaoyue, Tian Jing $\mathbb{D}$, and Wang Yingjie
}

Liaoning Key Laboratory of Advanced Measurement and Test Technology for Aviation Propulsion System, Shenyang Aerospace University, Shenyang 110136, China

Correspondence should be addressed to Tian Jing; jingtian@sau.edu.cn

Received 7 April 2021; Revised 4 June 2021; Accepted 22 October 2021; Published 17 November 2021

Academic Editor: Chengwei Fei

Copyright (C) 2021 Zhang Fengling et al. This is an open access article distributed under the Creative Commons Attribution License, which permits unrestricted use, distribution, and reproduction in any medium, provided the original work is properly cited.

\begin{abstract}
To further study the fault mechanism and fault features of rolling bearings, a two-DOF rolling bearing fault dynamic model with inner ring local defects considering the bearing radial clearance and time-varying displacement excitation is established based on Hertz contact theory. By comparing the simulated fault signal with bearing fault test data in the time domain and frequency domain, the accuracy of the established fault dynamic model is verified. Finally, the change rules of the characteristic parameters of the bearing inner ring fault signal, including effective value, absolute mean value, square root amplitude, peak value, kurtosis factor, pulse factor, peak factor, and shape factor, are simulated by the fault dynamic model. The results highlight that the proposed fault dynamic model is in good agreement with the experimental results. The model can simulate the fault signal characteristic parameters with the change of defect width, external load, and rotating speed effectively. The study in this paper is of engineering application value for bearing condition monitoring and fault diagnosis.
\end{abstract}

\section{Introduction}

Rolling bearing is an important part of various rotating machines. Bearing failures not only affect the normal operation of mechanical equipment but also cause catastrophic damages [1]. The condition monitoring and fault diagnosis for rolling bearings are hot topics of current research. It is of great significance to establish a reliable rolling bearing fault dynamic model for studying bearing condition monitoring and fault diagnosis $[2,3]$.

A lot of research has been carried out at home and abroad for the rolling bearing fault dynamic model. Patil et al. [4] established a dynamic model of rolling bearing based on Hertz contact theory and simulated the influence of the bearing outer ring local defect on bearing vibration. Patel et al. [5] studied the characteristics of deep groove ball bearing and built a two-DOF bearing dynamic model considering the shaft, cage, raceway, and ball quality. They also studied the vibration response of bearings with single fault and multiple faults on the inner and outer raceways of the bearing. Zhang et al. [6] built a two-DOF rolling bearing fault dynamic model with outer ring local defects, which considered the rolling shaft nonlinear bearing force and radial clearance. Guan et al. [7] set up three nonlinear dynamic fault models of rolling bearings with outer ring local defect, inner ring local defect, and the roller local defect, respectively, considering the bearing radial clearance, the nonlinear contact force of the roller and the raceway, and the varying compliance (VC) vibration. The correctness of these models is verified by simulation analysis results. To study the rolling bearing fault vibration characteristic, Zhang et al. [8] established a six-DOF bearing fault dynamic model. They simulated and analyzed the rolling bearing outer ring local defect fault, inner ring local defect fault, and roller local defect fault, respectively, by the Runge-Kutta numerical integration method. Their results are consistent with the experiment basically. Niu et al. [9] constructed the rolling ball bearing dynamic model with local surface defects based on the Gupta bearing model and analyzed its dynamic characteristic, which provided a theoretical foundation for 
rolling ball bearing fatigue damage analysis and fault quantitative diagnosis. Liu et al. [10] built the cylindrical roller bearing local defect fault dynamic model with nonideal Hertz line contact characteristic and time-varying displacement excitation. They also studied the effect of displacement excitation form and local defect size on the vibration characteristic of cylindrical roller bearing. It offered valuable conclusions for the incipient local defect fault dynamic analysis and fault diagnosis of rolling bearing. Yan et al. [11] instituted a twoDOF deep groove ball bearing local defect fault dynamic model, based on nonlinear Hertz contact deformation and elastic fluid lubrication theory. They also verified the accuracy of the model by comparing the simulation signal with the bearing fault test data and supplied a theoretical basis for bearing fault diagnosis. Niu et al. [1] proposed a dynamic model for roller bearings with roller defects. Jiang et al. [12] established an improved dynamic model to investigate the abrupt change of contact force and the corresponding vibration response of the bearing system. In order to investigate the vibration characteristics and stability due to fit clearance, Cao et al. [13] proposed a dynamic model of rotor-bearingpedestal system. The model applies time-varying displacement responses and Fourier spectra to analyze the vibration characteristics of the rotor system. Bourdon et al. [14] studied the effect of localized defects on the rotating speed fluctuation in a tapered roller bearing. The tool can distinguish speed changes from defects and working conditions. Li et al. [15] established a general dynamic model of rolling bearing rotor system and used a test rotor supported by two deep groove ball bearings for experimental verification. Yi et al. [16] proposed a model considering not only the high-speed effect of the rolling element such as centrifugal force and gyroscopic moment but also the degrees of freedom (DOFs) of all movable components. Qin et al. [17] proposed a new high-speed fault dynamic model of ACBB using a B-spline fitting displacement excitation method to represent the fault excitation.

Most of the research aimed at building the bearing fault dynamic models but lacked the change rules of bearing fault signal characteristic parameters [18-21]. In view of the above shortcomings, at first, the time-varying displacement excitation function is used to represent the fault in this paper according to the rolling bearing inner ring fault form; secondly, the rolling bearing fault dynamic model is built considering time-varying displacement excitation and bearing clearance. Then, the accuracy of the model is verified by comparing the model simulation results with the experimental results. Finally, when the inner ring fault occurs, the model is used to simulate the influence of the defect width, the external load, and the working speed on the bearing fault characteristic parameters. The research results can provide a theoretical reference for bearing condition monitoring and fault diagnosis.

\section{Bearing Fault Dynamic Model with Inner Ring Local Defects}

2.1. Simplified Bearing Model. The actual bearing structure is complex. To study the bearing vibration, the roller, the inner ring, and the outer ring can be simplified into a spring-mass system $[4,5]$, as shown in Figure 1 . The bearing fault dynamic model is established based on Hertz contact theory.

2.2. Local Defect Shape. This paper studies the incipient local defect fault of the bearing and uses the rectangle to represent the actual defect shape. During the incipient bearing fault, the width of the defect $W$ is small, so it does not touch the defect bottom when the rollers pass the defect. The maximum offset $H_{m}$ generated by the defect is smaller than the defect depth $H_{d}$. Figure 2 demonstrates the passing defect process of a roller, which is divided into three stages. During the first stage, the roller enters the defect from the beginning with the roller touching the left side of the defect, and the time-varying displacement $H_{t}$ is gradually increasing. During the second stage, the roller enters the defect completely with the roller touching both sides of the defect, and the time-varying displacement $H_{t}$ reaches the maximum. During the third stage, the roller leaves the defect with the roller touching the right side of the defect, and the timevarying displacement $H_{t}$ is dropping gradually. In brief, when the roller passes the defect, its time-varying displacement gradually increases and then decreases, so the roller time-varying displacement excitation model is expressed approximately by the sinusoidal function.

2.3. Time-Varying Displacement Excitation of Inner RingLocal Defect Fault. When the inner ring rotates with the working axis, both the position of the bearing inner ring defect and the time-varying displacement excitation change accordingly. The angular position relation of the roller and the defect is shown in Figure 3. The counterclockwise direction is the positive direction, and the inner ring angular velocity is set as $w_{i}$. The initial angular velocity of the first roller relative to the $X$ axis is $\theta_{b 0}$, and the angular velocity of the $i$ th roller relative to the $X$ axis at the time $t$ is $\theta_{b i}$. The initial angular velocity of the right side of the defect relative to the $X$ axis is $\theta_{f 0}$, and the angular velocity of the time $t$ relative to the $X$ axis is $\theta_{f t}$. The defect angle $\varphi_{f}$ is $2 W / d_{i}$, where $d_{i}$ is the diameter of the bearing inner raceway. According to the relationship of the inner ring defect and the roller motion, the time-varying displacement excitation $H_{t}$ generated by the roller passing the defect can be obtained as follows:

$$
H_{t}=\left\{\begin{array}{l}
H_{m} \sin \left(\frac{\pi}{\varphi_{f}}\left(\bmod \left(\theta_{f t}, 2 \pi\right)-\bmod \left(\theta_{b i}, 2 \pi\right)\right)\right), \\
0 \leq \bmod \left(\theta_{f t}, 2 \pi\right)-\bmod \left(\theta_{b i}, 2 \pi\right) \leq \varphi_{f}, \\
0,
\end{array}\right.
$$

where

$$
\begin{aligned}
& \theta_{f t}=\theta_{f 0}+w_{i} t, \\
& \theta_{b i}=\frac{2 \pi}{Z}(i-1)+w_{c} t+\theta_{b 0},
\end{aligned}
$$

in which $w_{c}$ is the speed of the bearing cage. 

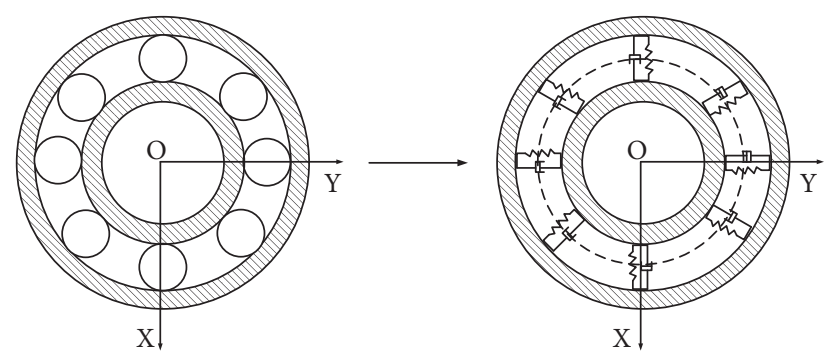

FIgURE 1: Simplified rolling bearing model.

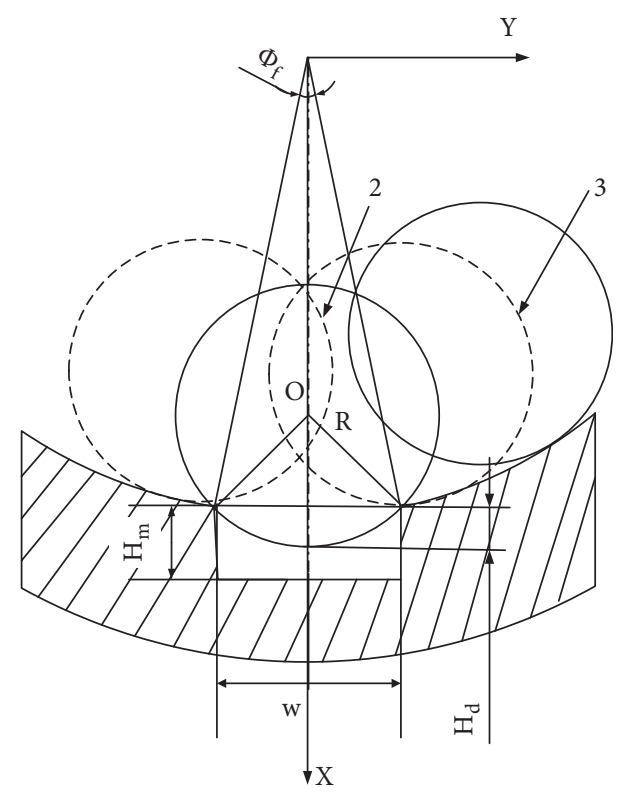

Figure 2: Process of roller passing defect.

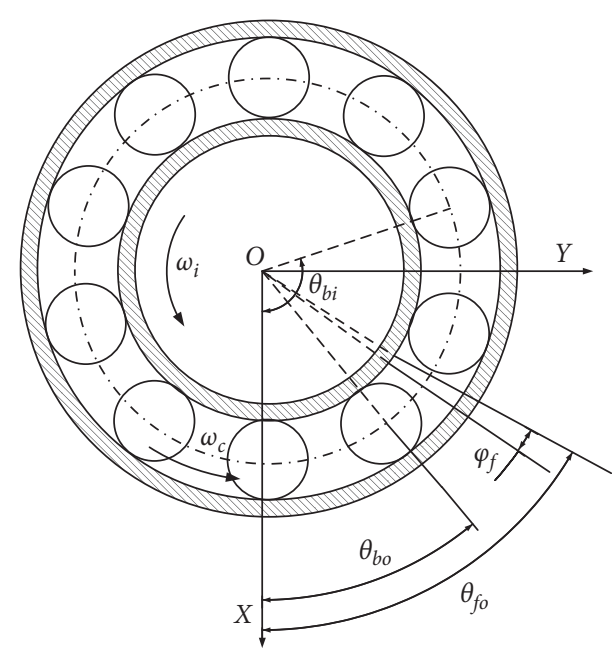

FIGURE 3: Angular position relation of roller and defect when bearing inner ring fault occurs.

2.4. Establishment of the Dynamic Differential Equation. The relationship between the bearing nonlinear load and displacement by Harris is expressed as follows:

$$
Q=K \delta^{n}
$$

For the deep groove ball bearing, $n=3 / 2$. When the bearing bears loads, the radial displacement between the two raceways is equal to the sum of the radial displacement between the two raceways and the roller, which is expressed as follows:

$$
\delta_{n}=\delta_{i}+\delta_{o}
$$

The total stiffness is written as

$$
K=\left(\frac{1}{\left(1 / K_{i}\right)^{1 / n}+\left(1 / K_{o}\right)^{1 / n}}\right)^{n},
$$

where $K_{i}$ is the contact stiffness of the roller and the inner race and $K_{o}$ is the contact stiffness of the roller and the outer race. For ball bearing,

$$
\begin{aligned}
& K_{i}=2.15 \times 10^{5} \sum \rho_{i}^{-1 / 2}\left(\delta_{i}^{*}\right)^{-3 / 2}, \\
& K_{o}=2.15 \times 10^{5} \sum \rho_{o}^{-1 / 2}\left(\delta_{o}^{*}\right)^{-3 / 2},
\end{aligned}
$$

where $\delta_{i}^{*}$ is the contact parameter when the inner raceway dimension is 1 and $\delta_{o}^{*}$ is the contact parameter when the outer raceway dimension is $1 . \sum \rho_{i}$ is the curvature function of the inner raceway. $\sum \rho_{o}$ is the curvature function of the outer raceway.

The bearing total contact stiffness $K$ can be calculated by formula (5). The displacement of the inner ring of the bearing in the $X$ and $Y$ directions is $x_{i}$ and $y_{i}$, as shown in Figure 4 . The radial displacement $\delta$ of the inner ring relative to a roller is expressed as follows:

$$
\delta=x_{i} \cos \theta_{\mathrm{bi}}+y_{i} \sin \theta_{\mathrm{bi}}-C_{r}
$$

where $C_{r}$ is the radial clearance. For the deep groove ball bearing, the radial contact force $Q_{i}$ of each roller can be calculated by the following formula:

$$
Q_{i}=K\left[x_{i} \cos \theta_{b i}+y_{i} \sin \theta_{b i}-\left(C_{r}+H_{t}\right)\right]^{3 / 2},
$$

where $F_{I X}$ and $F_{I Y}$ are the sum of the component forces of the radial contact force $Q_{i}$ of each ball in the $X$ and $Y$ directions and are written as the following formulas, respectively:

$$
\begin{gathered}
F_{I X}=K \sum_{i=1}^{Z}\left[x_{i} \cos \theta_{b i}+y_{i} \sin \theta_{b i}-\left(C_{r}+H_{t}\right)\right]^{3 / 2} \cos \theta_{b i} \\
F_{I Y}=K \sum_{i=1}^{Z}\left[x_{i} \cos \theta_{b i}+y_{i} \sin \theta_{b i}-\left(C_{r}+H_{t}\right)\right]^{3 / 2} \sin \theta_{b i} .
\end{gathered}
$$

When the rolling bearing is working, the outer ring is generally fixed in the bearing housing. Due to the high rigidity of the bearing housing and the small vibration of the bearing outer ring, only the vibration of the inner ring is considered, and the differential equation of the bearing fault dynamic with two DOFs is established as shown in the following formula [11]: 


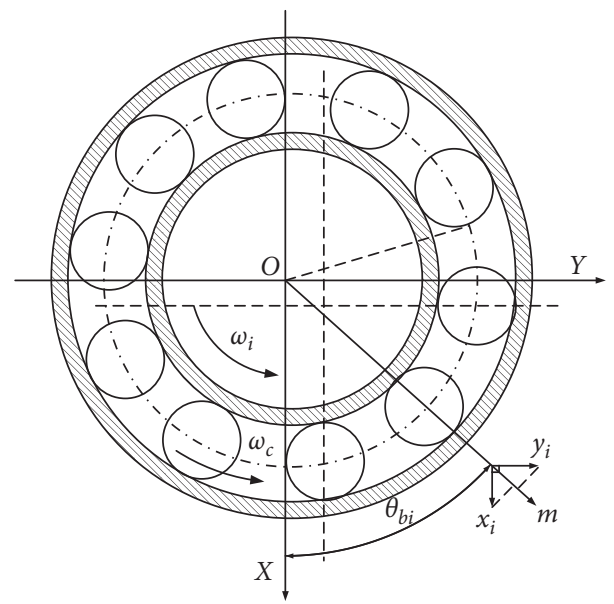

FIgURE 4: Deflection of bearing inner ring in $X$ and $Y$ directions.

$$
\begin{aligned}
& M_{i} \frac{d^{2} x_{i}}{d t^{2}}+C \frac{d x_{i}}{d t}+K \sum_{i=1}^{Z}\left[x_{i} \cos \theta_{b i}+y_{i} \sin \theta_{b i}-\left(C_{r}+\lambda_{1} H_{t}\right)\right]^{3 / 2} \cos \theta_{b i}=F_{I X}+F_{i} \cos \left(w_{i} t\right), \\
& M_{i} \frac{d^{2} y_{i}}{d t^{2}}+C \frac{d y_{i}}{d t}+K \sum_{i=1}^{Z}\left[x_{i} \cos \theta_{b i}+y_{i} \sin \theta_{b i}-\left(C_{r}+\lambda_{1} H_{t}\right)\right]^{3 / 2} \sin \theta_{b i}=F_{I Y}+F_{i} \sin \left(w_{i} t\right),
\end{aligned}
$$

where $M_{i}$ is the mass of the inner ring and $\lambda_{1}$ is the switch value judging whether the roller passes the defect or not. If it is "pass," $\lambda_{1}=1$; otherwise, $\lambda_{1}=0$. $C$ is the bearing inner ring equivalent damping in the $X$ and $Y$ directions. $F_{I X}$ and $F_{I Y}$ are the external loads acting on the inner ring in the $X$ and $Y$ directions, respectively. $F_{i}$ is the inner ring centrifugal force by eccentricity, and $w_{i}$ is the inner ring angular velocity.

The bearing cross stiffness is negligible [22], so the bearing stiffness $K_{I X}$ and $K_{I Y}$ in the $X$ and $Y$ directions can be approximated by the deviation of formulas (9) and (10) for $x$ and $y$.

$$
\left[\begin{array}{c}
K_{I X} \\
K_{I Y}
\end{array}\right]=\left[\begin{array}{c}
\frac{\partial Q_{x}}{\partial x} \\
\frac{\partial Q_{y}}{\partial y}
\end{array}\right]=\frac{3}{2} K \sum_{i=1}^{Z}\left[x_{i} \cos \theta_{b i}+y_{i} \sin \theta_{b i}-\left(C_{r}+\lambda_{1} H_{t}\right)\right]^{1 / 2}\left[\begin{array}{c}
\cos ^{2} \theta_{b i} \\
\sin ^{2} \theta_{b i}
\end{array}\right]
$$

In summary, the steps to solve the dynamic equation of ball bearing in this paper are shown in Figure 5, as follows.

\section{Experimental Verification}

3.1. Acquisition of Bearing Test Data. The correctness of the bearing fault dynamic model is verified by comparing the bearing fault test data of Case Western Reserve University with model simulation results. The bearing fault test rig is shown in Figure 6. It includes a 2-horsepower motor, a torque sensor, a power meter, and an electronic control unit. The bearing outer ring is fixed, and the inner ring supports the motor shaft. The vibration signals are collected by acoustic sensors, which are mounted on the motor housing at 6 o'clock. The sampling frequency is $12000 \mathrm{~Hz}$, and the motor spindle speed is $1797 \mathrm{r} / \mathrm{min}$.
The test bearing is a deep groove bearing (Model: 62052RS JEM SKF), and the structural parameters are shown in Table 1. A single point fault is set on the bearing inner ring by the spark erosion technique. The defect width is $0.1778 \mathrm{~mm}$, and the defect depth is $0.2794 \mathrm{~mm}$.

The local defect fault feature frequency formula of the bearing inner ring [23] is expressed as follows:

$$
f_{o}=\frac{f}{2}\left(1+\frac{d}{d_{m}} \cos \alpha\right) Z,
$$

where $f$ is the bearing inner ring frequency, $d$ is the roller diameter, $d_{m}$ is the bearing pitch diameter, and $\alpha$ is the contact angle between the roller and the inner or outer ring. By calculation, the inner ring fault feature frequency $f_{o}$ is $162.8 \mathrm{~Hz}$, and the inner ring rotating frequency $f$ is $29.9 \mathrm{~Hz}$. 


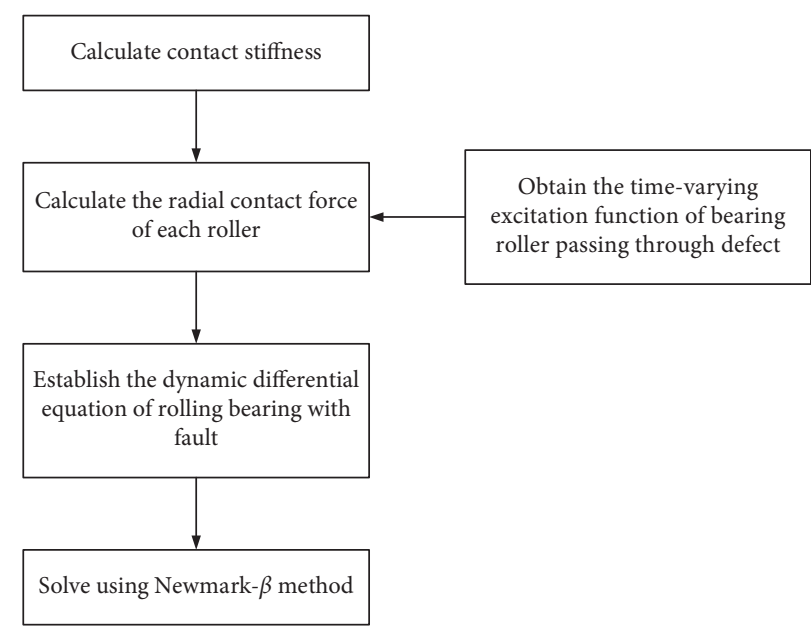

Figure 5: Calculation flowchart of the proposed dynamic model.

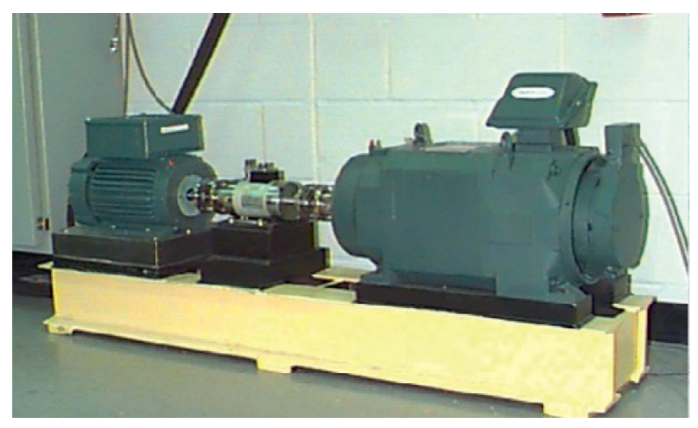

(a)

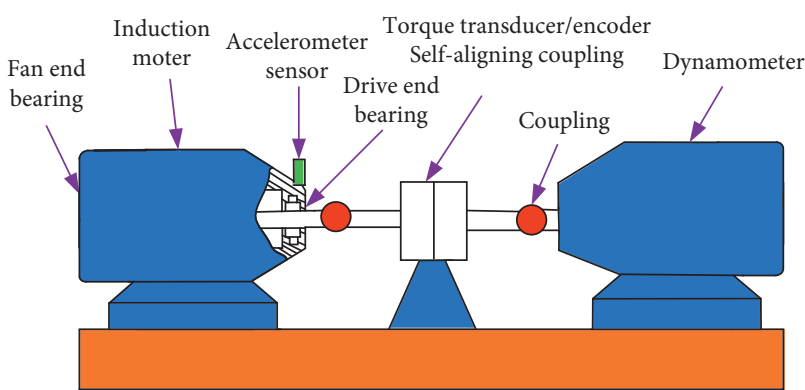

(b)

Figure 6: Bearing fault test rig. (a) Experimental setup. (b) Schematic drawing of the experimental setup.

TABLE 1: 6205-2RS JEM SKF bearing structural parameters.

\begin{tabular}{lc}
\hline Parameter & Numerical value \\
\hline Bearing inner race diameter $d_{i}(\mathrm{~mm})$ & 25 \\
Bearing outer race diameter $d_{o}(\mathrm{~mm})$ & 52 \\
Pitch diameter $d_{m}(\mathrm{~mm})$ & 38.5 \\
Roller diameter $d(\mathrm{~mm})$ & 8 \\
Number of balls $Z$ & 9 \\
Contact angle $\alpha$ & 0 \\
Radial clearance $C_{r}(\mu \mathrm{m})$ & 20 \\
\hline
\end{tabular}

The parameters are set according to Table 1 . The bearing inner ring structural damping is $200 \mathrm{Ns} / \mathrm{m}$. The radial load $F_{I X}$ in the $X$ direction is $300 \mathrm{~N}$, the radial load $F_{I Y}$ in the $Y$ direction is $0 \mathrm{~N}$, and the eccentric distance of the inner ring is $0.005 \mathrm{~mm}$. The fault dynamic model is solved by theNewmark $-\beta$ method. The time step is $0.0001 \mathrm{~s}$, the initial displacement, the initial speed, and the initial acceleration in $X$ direction and $Y$ direction are both zero.

\subsection{Inner Ring Fault Simulation and Experimental} Verification. When the bearing inner ring has local defect, the load at the defect changes periodically because of the defect rotating with the bearing inner ring, and the bearing frequency plays the role of frequency modulation. The spectrogram of the inner ring fault signal is mainly described as the inner ring fault feature frequency and its frequency multiplication, The the inner ring fault feature frequency is the center frequency, while the rotating frequency and its frequency multiplication are the side frequencies.

The time-domain signal of the bearing inner ring fault test data is shown in Figure 7(a). The frequency spectrum signal after noise reduction is shown in Figure $7(\mathrm{~b})$. Figure 8 (a) shows the simulation signal of the bearing inner ring fault model in the time domain, and the frequencydomain simulation signal is shown in Figure 8(b). From Figures 7(b) and 8(b), it can be seen that the fault feature frequency $f_{i}$ and its doubling frequency $2 f_{i}$ are clear, with $f_{i}$ as the center, $f$ as the rotating frequency, and its frequency multiplication $2 f$ as the side band. Experimental results and simulation results are in good agreement. The fault feature frequency $f_{i}=162.8 \mathrm{~Hz}$ in the simulation signal frequency spectrum, which is consistent with the theoretical value. The fault feature frequency in the experimental signal frequency spectrum $f_{i}=161.7 \mathrm{~Hz}$, which is basically equal to the simulation result. They are a little different because there is a slip phenomenon when the bearing works and an error in the bearing speed measurement. Therefore, the accuracy of the inner ring fault dynamic model is verified by comparing the simulation results with the experimental results. 


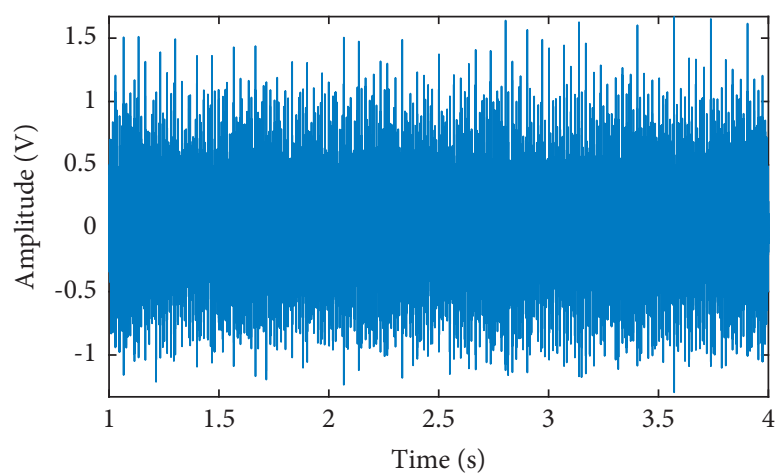

(a)

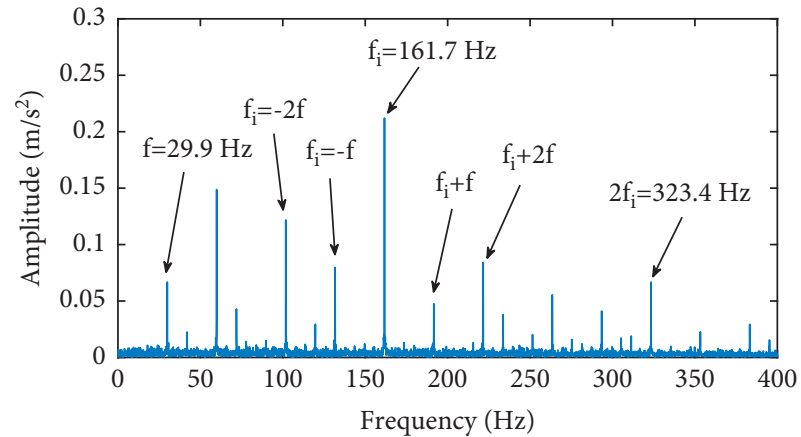

(b)

Figure 7: Test signal of bearing with inner ring fault. (a) Test signal in time domain. (b) Test signal in frequency domain after noise reduction.

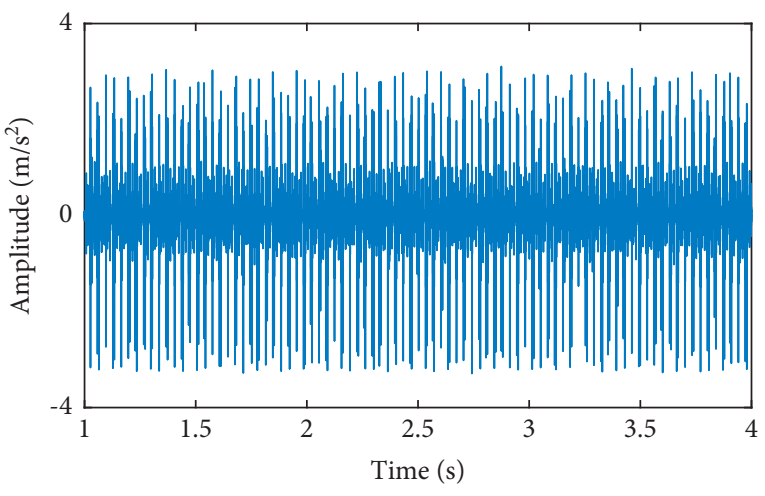

(a)

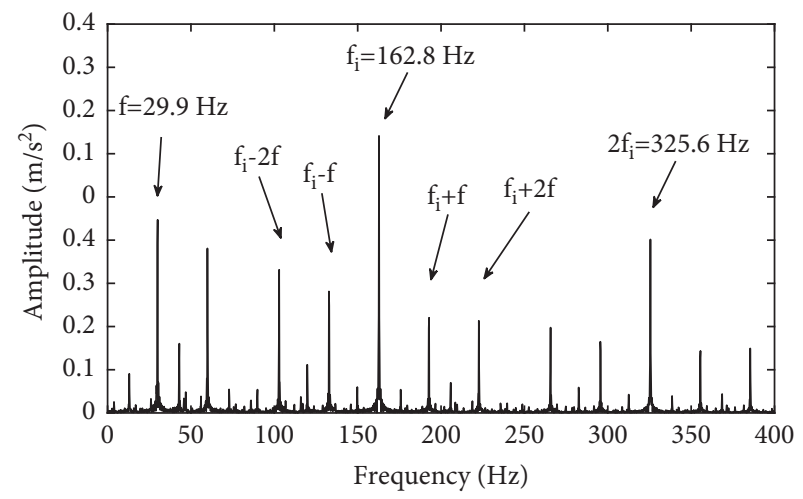

(b)

FIGURE 8: Simulation signal of the bearing inner ring fault model in (a) time domain and (b) frequency domain.

\section{Characteristic Parameter Simulation and Analysis of Bearing Inner Ring Fault Signals}

4.1. Characteristic Parameters of Fault Signals. In the early stage of bearing local defect fault, the defect size is very small, but the defect size expands gradually with the operation of the bearing until a serious fault occurs. In addition, the radial load and working speed of the bearing will also change. Therefore, it is necessary to study the fault signal characteristics of the bearing under different defect widths, radial loads, and working speeds and get the corresponding change law, which can provide a reference for bearing condition monitoring and fault diagnosis.

For the bearing fault signal, the statistical parameters which can reflect the fault characteristics are usually selected to describe its characteristics, including dimensional characteristic parameters and dimensionless characteristic parameters. Peak value $X_{\max }$, absolute mean value $X_{a}$, effective value $X_{\text {rms }}$, and square root amplitude $X r$ are dimensional characteristic parameters. $x(k), k=1 \ldots N$ is a discrete signal sequence, and the calculation formulas of the above characteristic parameters are shown in Table 2.

The peak value can reflect the impact force of bearing fault, and the greater the impact force is, the greater the peak value is. It is suitable for the fault diagnosis of bearing surface
TABLE 2: Calculation formulas of dimensional characteristic parameters.

\begin{tabular}{lc}
\hline $\begin{array}{l}\text { Dimensional characteristic } \\
\text { parameters }\end{array}$ & Calculation formula \\
\hline Peak value $X_{\max }$ & $X_{\max }=\max (x(k))$ \\
Absolute mean value $X_{a}$ & $X_{a}=1 / N \sum_{k=1}^{N}|x(k)|$ \\
Effective value $X_{\text {rms }}$ & $X_{\text {rms }}=\sqrt{1 / N \sum_{k=1}^{N} x^{2}(k)}$ \\
Square root amplitude $X_{r}$ & $X_{r}=\left(1 / N \sum_{k=1}^{N} \sqrt{|x(k)|}\right)^{2}$ \\
\hline
\end{tabular}

pitting defects, especially in the early stage of bearing fault; the peak value is very sensitive to the fault. The absolute mean value, square root amplitude, and effective value can reflect the magnitude of signal energy. The greater the energy value is, the stronger the bearing fault vibration is, and the more serious the fault is.

Kurtosis factor $K_{v}$, pulse factor $I_{f}$, peak factor $C_{f}$, and shape factor $S_{f}$ are the dimensionless characteristic parameters. The calculation formulas are shown in Table 3.

$\beta$ is the kurtosis, $\sigma$ is the standard deviation, and $\mu$ is the mean value.

$$
\beta=\frac{1}{N} \sum_{K=1}^{N}(x(k)-\mu)^{4} .
$$


TABLE 3: Calculation formulas of dimensionless characteristic parameters.

\begin{tabular}{lr}
\hline Dimensionless characteristic parameters & Calculation formula \\
\hline Kurtosis factor $K_{v}$ & $K_{v}=\beta / \sigma^{4}$ \\
Pulse factor $I_{f}$ & $I_{f}=X_{\max } / X_{a}$ \\
Peak factor $C_{f}$ & $C_{f}=X_{\max } / X_{\mathrm{rms}}$ \\
Shape factor $S_{f}$ & $S_{f}=X_{\mathrm{rms}} / X_{a}$ \\
\hline
\end{tabular}

The kurtosis factor is the ratio of the kurtosis $\beta$ to the fourth power of the standard deviation $\sigma^{4}$. The vibration signal of bearings without faults satisfies a normal distribution, and the kurtosis factor is 3 . The kurtosis factor of bearings with faults is larger than 3 . When the bearing fault occurs, the components with large amplitude in the signal increase because of the shock pulse in the fault signal, and the two ends of the probability density distribution function are upwarped. So, the kurtosis factor of fault bearing is greater than 3 . The kurtosis factor is very sensitive to the early fault of bearing.

The pulse factor is the ratio of the peak value to the absolute average value, which can judge whether there is an impact component in the signal and has a good monitoring effect on pitting, scratch, and other faults. The peak factor is the ratio of the peak value to the effective value, which is sensitive to the impact vibration caused by surface peeling and scratch. The shape factor is the ratio of effective value and absolute average value, which has a certain monitoring effect on bearing pitting and wear fault.

Taking the bearing inner ring local defect fault as an example, the influence of defect width, external load, and working speed on the characteristic parameters of the bearing fault signal is studied in the following paragraphs.

\subsection{Influence of Defect Width on Characteristic Parameters of} Bearing Inner Ring Fault Signals. Considering the rotation speeds, the change rule of characteristic parameters of the inner ring fault signal with the defect width is studied. The radial load is kept constant at $300 \mathrm{~N}$, the defect width is from 0 to $2 \mathrm{~mm}$, the defect increment is $0.1 \mathrm{~mm}$, the bearing working speed is from $500 \mathrm{r} / \mathrm{min}$ to $15500 \mathrm{r} / \mathrm{min}$, and the rotational speed increment is $1000 \mathrm{r} / \mathrm{min}$.

Figures 9(a) 9(c) show the change law of the effective value, square root amplitude, and absolute mean value with defect width. When the rotation speed is low, the three characteristic parameters increase gradually with the defect width increasing and remain unchanged when the defect width reaches a certain value. When the rotation speed is high, the parameters increase continuously. It is because the increase of the defect width causes the increase of large amplitude components and the signal energy of the fault signal. Figure 9(d) shows the change law of the peak value with defect width. In the early stage of the fault, the peak value increases with the increase of the defect width. In the later stage of the fault, although the defect width continues to increase, the peak value does not increase and fluctuates irregularly due to the limitation of the structural characteristics of the bearing itself. From the above analysis, in the early stage of bearing failure, the dimensional characteristic parameters are more sensitive to the change of defect width, and there is no significant difference in the sensitivity of dimensional characteristic parameters to the change of defect width under different speeds except for the very low working speed.

Figures $9(\mathrm{e})-9(\mathrm{~h})$ show the change law of the kurtosis factor, pulse factor, peak factor, and shape factor with defect width. At low speed, the dimensionless characteristic parameters increase rapidly with the increase of defect width, then decrease slightly, and then remain unchanged; at high speed, with the increase of defect width, the dimensionless characteristic parameters first increase and then gradually decrease. From the calculation formula of dimensionless characteristic parameters, the reason for the above variation is that the relative growth rate of dimensional characteristic parameters on the numerator and denominator of dimensionless characteristic parameters is different with the increase of defect width in different periods of bearing failure. As shown in Table 4, the dimensionless characteristic parameters increase firstly and then decrease with the expansion of defects.

In addition, it can be found that the dimensionless characteristic parameters at low speed are significantly larger than those at high speed, and with the increase of working speed, the speed of dimensionless characteristic parameters with the increase of defect width gradually decreases. It can be seen from the above analysis that, in the early stage of bearing fault, the dimensionless parameters are very sensitive to the change of defect width, but with the increase of bearing working speed, the statistical characteristics of the fault signal are changed, and the sensitivity of dimensionless parameters to the change of defect width is gradually reduced. In practice, by monitoring the characteristic parameters of the bearing vibration signal, the bearing fault can be predicted, and the development trend of the bearing fault can be reflected.

\subsection{Influence of Working Speed on Characteristic Parameters} of Inner Ring Fault Signals. Considering the defect width, the change rule of characteristic parameters of the inner ring fault signal with the working speed is also studied. The external load is $300 \mathrm{~N}$, the working speed is $500 \mathrm{r} / \mathrm{min}$ $\sim 15500 \mathrm{r} / \mathrm{min}$, the speed increment is $500 \mathrm{r} / \mathrm{min}$, the defect width is $0 \sim 2 \mathrm{~mm}$, and the defect width increment is $0.2 \mathrm{~mm}$. When the defect width is 0 , the bearing is normal without any fault.

Figures 10(a) 10(c) show the change law of the effective value, square root amplitude, and absolute mean value with the working speed. In the early stage of bearing fault, the parameters increase slowly with the increase of the rotating 


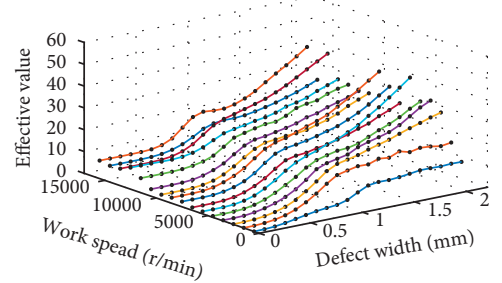

(a)

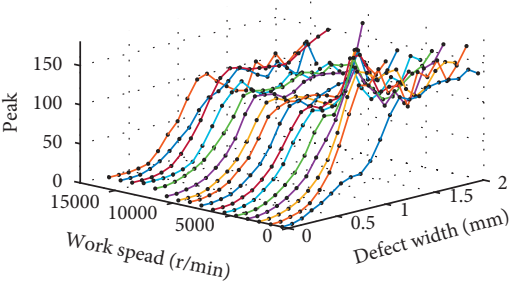

(d)

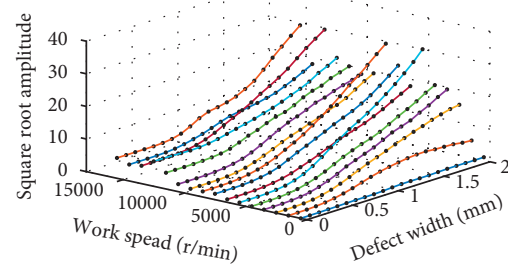

(b)

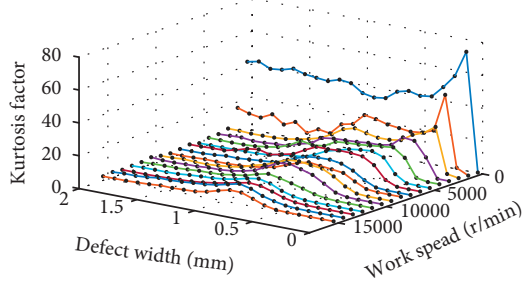

(e)

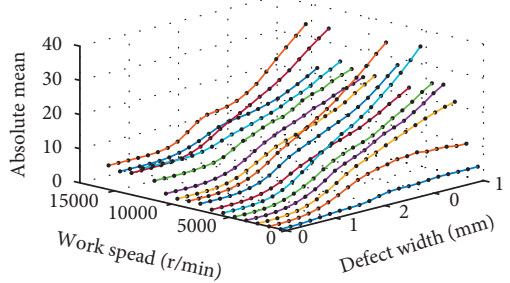

(c)

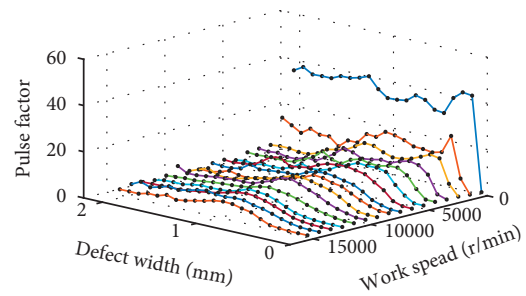

(f)

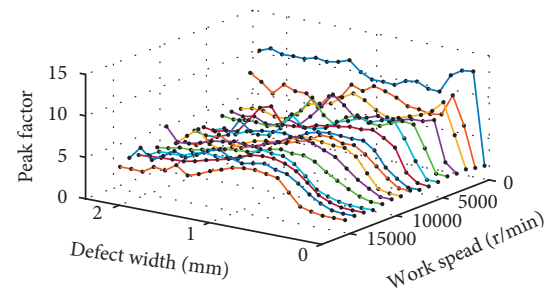

(g)

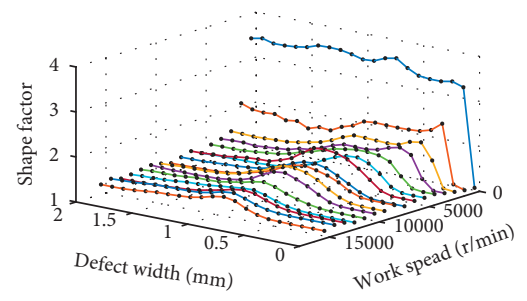

(h)

FIGURE 9: Effect of defect width on characteristic parameters under different working speeds. (a) Effective value. (b) Square root amplitude. (c) Absolute mean value. (d) Peak value. (e) Kurtosis factor. (f) Pulse factor. (g) Peak factor. (h) Shape factor.

TABLE 4: Relative growth speed of characteristic parameters in different periods.

\begin{tabular}{lccc}
\hline \multicolumn{1}{c}{ Early fault } & \multicolumn{2}{c}{ Late fault } \\
\hline$\beta \uparrow>\sigma^{4} \uparrow$ & $K_{v} \uparrow$ & $\beta \uparrow<\sigma^{4} \uparrow$ & $K_{v} \downarrow$ \\
$X_{\max } \uparrow>X_{a} \uparrow$ & $I_{f} \uparrow$ & $X_{\max } \uparrow<X_{a} \uparrow$ & $I_{f} \downarrow$ \\
$X_{\max } \uparrow>X_{\mathrm{rms}} \uparrow$ & $C_{f} \uparrow$ & $X_{\max } \uparrow<X_{\mathrm{rms}} \uparrow$ & $C_{f} \downarrow$ \\
$X_{\mathrm{rms}} \uparrow>X_{a} \uparrow$ & $S_{f} \uparrow$ & $X_{\mathrm{rms}} \uparrow<X_{a} \uparrow$ & $S_{f} \downarrow$ \\
\hline
\end{tabular}

speed and then remain unchanged; when the defect width is large, the parameters increase rapidly with the increase of the rotating speed. Figure 10(d) shows the change law of the peak with the working speed. When the defect width is small, the peak value increases slowly and then remains unchanged. When the defect width is large, the peak has irregular fluctuations. It can be seen from above that, in the early stage of faults, the dimensional characteristic parameters are insensitive to the rotation speeds.

Figures $10(\mathrm{e})-10(\mathrm{~h})$ show the change of the kurtosis factor, pulse factor, peak factor, and shape factor with the working speeds. For the normal bearing, the dimensionless characteristic parameters are basically unchanged. Because there are no impact components in the signal, the dimensionless characteristic parameters are only sensitive to the fault signal impact components. For the bearing inner ring fault, the dimensionless characteristic parameters reduce rapidly from the initial maximum value and then remain unchanged. The larger the defect width is, the faster the decrease in parameters will be. In practice, the bearing fault can be predicted by monitoring the change of dimensionless characteristic parameters of mechanical equipment bearing vibration signal with speed, which has a certain practical significance.

\subsection{Influence of External Load Size on Characteristic Pa-} rameters of Inner Ring Fault Signals. The change law of characteristic parameters of the inner ring fault signal with the external load size is finally studied. The external load is $200 \mathrm{~N} \sim 1500 \mathrm{~N}$, the external load increment is $100 \mathrm{~N}$, the defect width is 0 to $2 \mathrm{~mm}$, the defect increment is $0.1 \mathrm{~mm}$, and the rotation speed is $8000 \mathrm{r} / \mathrm{min}$.

Figures 11(a)-11(d) show the change of the effective value, square root amplitude, absolute mean value, and peak value with the defect widths. Under different external loads, the dimensional characteristic parameters gradually increase. The larger the external load is, the faster the increase in parameters will be. When the external load reaches a certain value, the bearing capacity reaches the upper limit, and the parameter values tend to be stable. It can be seen from the analysis that, in the incipient bearing fault, the dimensional characteristic parameters are sensitive to the defect width, and the sensitivity is not significant under different external loads.

Figures 11(e)-11(h) show the change of the kurtosis factor, pulse factor, peak factor, and shape factor with the 


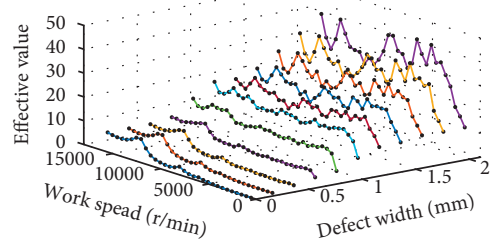

(a)

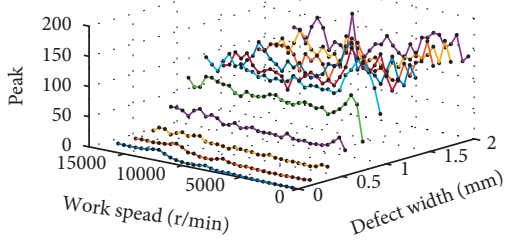

(d)

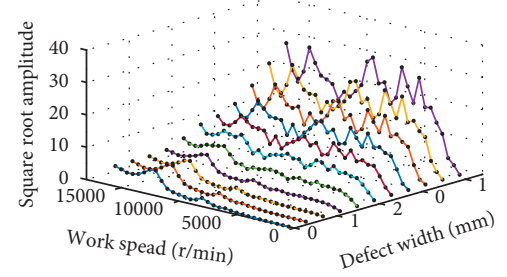

(b)

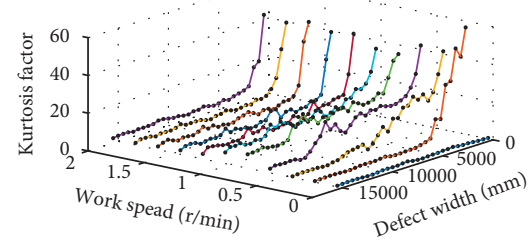

(e)

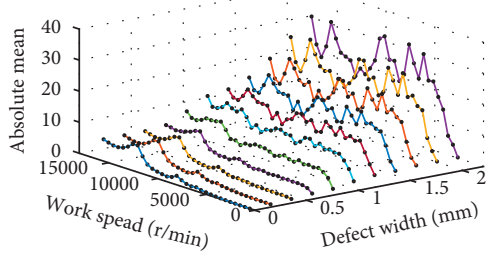

(c)

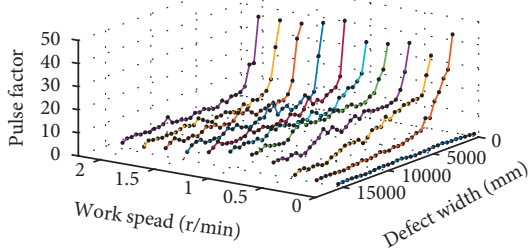

(f)

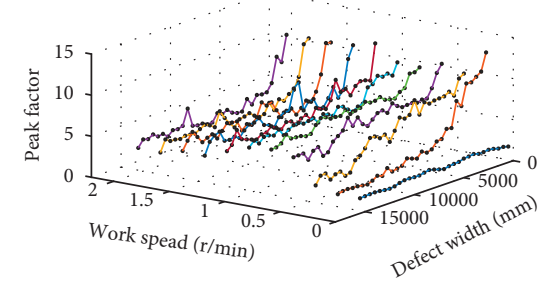

(g)

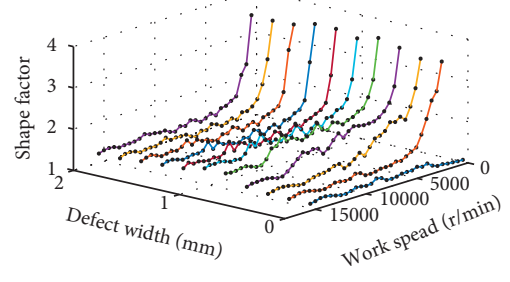

(h)

FIGURE 10: Effect of working speed on characteristic parameters under different defect widths. (a) Effective value. (b) Square root amplitude. (c) Absolute mean value. (d) Peak value. (e) Kurtosis factor. (f) Pulse factor. (g) Peak factor. (h) Shape factor.

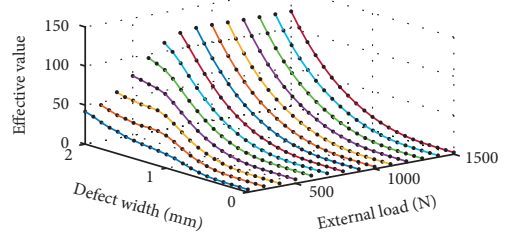

(a)

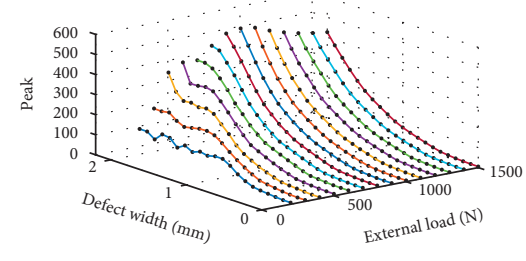

(d)

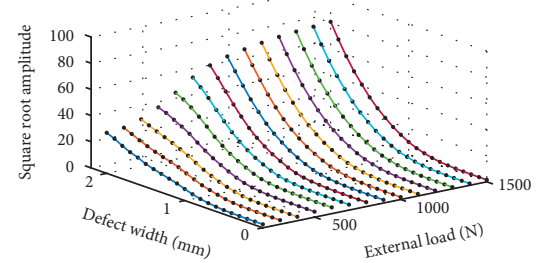

(b)

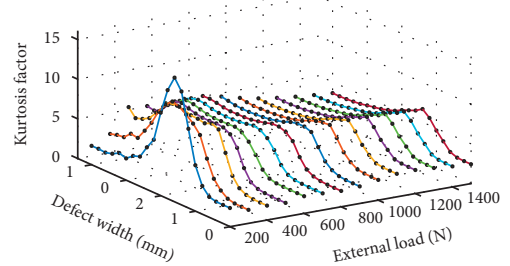

(e)

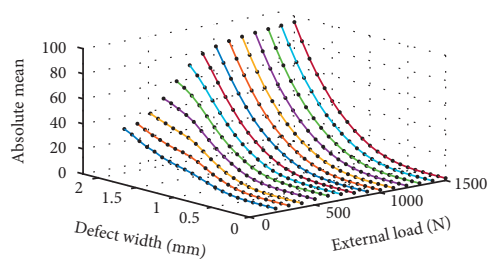

(c)

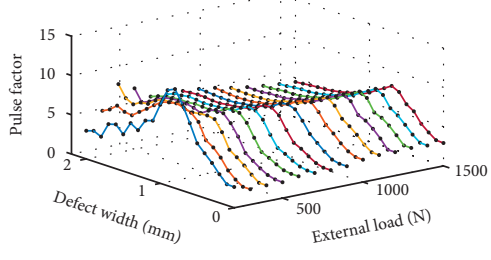

(f)

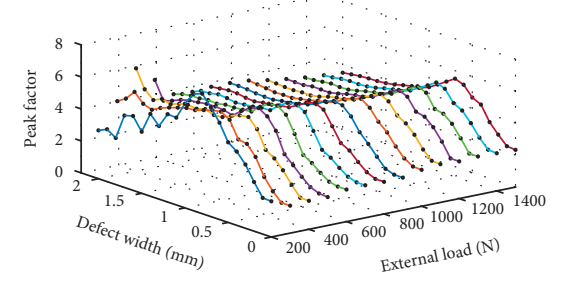

(g)

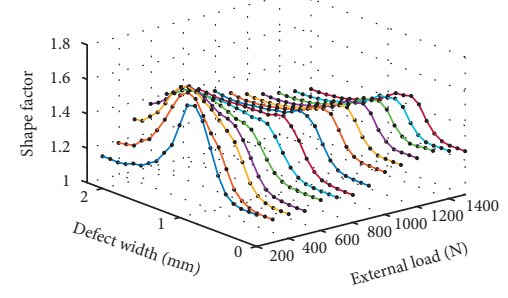

(h)

FIGURE 11: Effect of defect width on characteristic parameters under different external loads. (a) Effective value. (b) Square root amplitude. (c) Absolute mean value. (d) Peak value. (e) Kurtosis factor. (f) Pulse factor. (g) Peak factor. (h) Shape factor. 
defect width. The parameters increase firstly and then gradually decrease. As the external load increases, all the parameter values decrease. It is because the increase of the external load suppresses the fault impact vibration and reduces the dimensionless parameter values.

\section{Conclusions}

This paper uses the time-varying displacement excitation function to indicate faults aiming at the bearing inner ring fault form. Then, the rolling bearing fault dynamic model is built considering the time-varying displacement excitation and bearing clearance. At last, the accuracy of the model is verified by comparing the model computing results with the experimental results. The conclusions are as follows:

(1) The fault dynamic model of bearing inner ring with local defect is established, which considers the timevarying displacement excitation and radial clearance. By comparing the bearing fault test data, it is verified that the time-varying displacement excitation established in this paper can accurately simulate the bearing fault excitation, and the bearing fault dynamic model can accurately simulate the bearing fault characteristics.

(2) In the incipient bearing inner ring fault, the dimensional characteristic parameters of the fault signal are sensitive to defect width and increase with defect width increasing. The sensitivity is not significant at different working speeds.

(3) The dimensionless characteristic parameters of the fault signal increase first and then decrease with the defect width increasing. In the incipient fault, those parameters are very sensitive, but as the bearing rotation speed increases, the sensitivity decreases.

(4) For normal bearing, with the working speed increasing, the dimensional characteristic parameters increase slowly, and the dimensionless characteristic parameters remain unchanged. In the incipient inner ring fault, the dimensional characteristic parameters change slowly and are insensitive with the working speed increasing, but the dimensionless characteristic parameters reduce rapidly from the initial maximum value and are very sensitive.

(5) In the incipient inner ring fault, there is no significant difference in the sensitivity of dimensional characteristic parameters to the change of defect width under different external loads. The increase of the external load reduces the sensitivity of the dimensionless characteristic parameters to the defect width.

The efforts of this study provide a useful insight to adopt a dynamic model to evaluate and monitor the health condition of bearings in an aeroengine or other rotating machinery. In future, the method in this paper may also be used in other kinds of bearings, such as intershaft bearings in aeroengines.

\section{Data Availability}

All data generated or analyzed during this study are included in this article.

\section{Conflicts of Interest}

The authors declare that they have no conflicts of interest.

\section{Acknowledgments}

This wok was supported by the National Natural Science Foundation of China (Grant no. 12172231), Natural Science Foundation of Liaoning Province (Grant no. 2020-BS-174), Research Start-up Funding of Shenyang Aerosapce University (Grant no. 120421004), and Project of Liaoning Provincial Department of Education (Grant nos. JYT2020033 and JYT2020019).

\section{References}

[1] L. Niu, H. Cao, H. Hou, B. Wu, Y. Lan, and X. Xiong, "Experimental observations and dynamic modeling of vibration characteristics of a cylindrical roller bearing with roller defects," Mechanical Systems and Signal Processing, vol. 138, Article ID 106553, 2020.

[2] J. Liu, C. Tang, H. Wu, Z. Xu, and L. Wang, “An analytical calculation method of the load distribution and stiffness of an angular contact ball bearing," Mechanism and Machine Theory, vol. 142, Article ID 103597, 2019.

[3] J. Liu, Y. Xu, and G. Pan, "A combined acoustic and dynamic model of a defective ball bearing," Journal of Sound and Vibration, vol. 501, no. 1, Article ID 116029, 2021.

[4] M. S. Patil, J. Mathew, P. K. Rajendrakumar, and D. Sandeep, "A theoretical model to predict the effect of localized defect on vibrations associated with ball bearing," International Journal of Mechanical Sciences, vol. 52, no. 5, pp. 1193-1201, 2010.

[5] V. N. Patel, N. Tandon, and R. K. Pandey, "A dynamic model for vibration studies of deep groove ball bearings considering single and multiple defects in races," Journal of Tribology, vol. 132, no. 4, pp. 041101-0411101-10, 2010.

[6] Y. Zhang, J. Chen, and L. Tang, "Nonlinear dynamic characteristics of rolling bearings considering local defects in outer ring," Chinese Journal of Aeronautics, vol. 30, no. 4, pp. 751-756, 2009.

[7] Z. Guan, H. Zheng, and Y. Wang, "Dynamic modeling and Simulation of local damage of rolling bearing," Journal of Vibration, Measurement \& Diagnosis, vol. 32, no. 6, pp. 950-955, 2012.

[8] J. Zhang, Z. Wang, and Y. Lu, "Modeling and analysis of rolling bearing fault engineering based on nonlinear dynamics," Journal of Vibration and Shock, vol. 29, no. 11, pp. 30-34, 2010.

[9] L. Niu, H. Cao, and Z. Ho, "Dynamic modeling of rolling ball bearing with localized surface defects considering three dimensional motions and relative slippage," Journal of $\mathrm{Me}$ chanical Engineering, vol. 51, no. 19, pp. 53-59, 2015.

[10] J. Liu, Y. Shao, and X. Qin, "Dynamic modeling on localized defect of cylindrical roller bearing based on non-hertz line contact characteristics," Journal of Mechanical Engineering, vol. 50, no. 1, pp. 91-97, 2014.

[11] C. Yan, H. Yuan, X. Wang, X. Wu, and Y. Wei, "Dynamics modeling on local defect of deep groove ball bearing under 
point contact elasto-hydrodynamic lubrication condition," Journal of Vibration and Shock, vol. 35, no. 14, pp. 61-70, 2016.

[12] Y. Jiang, W. Huang, J. Luo, and W. Wang, "An improved dynamic model of defective bearings considering the threedimensional geometric relationship between the rolling element and defect area," Mechanical Systems and Signal Processing, vol. 129, pp. 694-716, 2019.

[13] H. Cao, F. Shi, Y. Li, B. Li, and X. Chen, "Vibration and stability analysis of rotor-bearing-pedestal system due to clearance fit," Mechanical Systems and Signal Processing, vol. 133, Article ID 106275, 2019.

[14] A. Bourdon, S. Chesné, H. André, and D. Rémond, "Reconstruction of angular speed variations in the angular domain to diagnose and quantify taper roller bearing outer race fault," Mechanical Systems and Signal Processing, vol. 120, pp. 1-15, 2019.

[15] Y. Li, H. Cao, and K. Tang, "A general dynamic model coupled with EFEM and DBM of rolling bearing-rotor system," Mechanical Systems and Signal Processing, vol. 134, Article ID 106322, 2019.

[16] Q. Yi, C. Li, X. Wu, and Y. Wang, "Multiple-degree-offreedom dynamic model of rolling bearing with a localized surface defect," Mechanism and Machine Theory, vol. 154, Article ID 104047, 2020.

[17] Y. Qin, C. Li, F. Cao, and H. Chen, "A fault dynamic model of high-speed angular contact ball bearings," Mechanism and Machine Theory, vol. 143, Article ID 103627, 2020.

[18] B. Fang, L. Zhang, and T. Qu Xing, "Dynamic modeling and experiment of angular contact ball bearings," Transactions of the Chinese Society for Agricultural Machinery, vol. 43, no. 6, pp. 215-219, 2012.

[19] J. Liu and Y. Shao, "Dynamic modeling for rigid rotor bearing systems with a localized defect considering additional deformations at the sharp edges," Journal of Sound and Vibration, vol. 398, 2017.

[20] S. Khanam, N. Tandon, and J. K. Dutt, "Multi-event excitation force model for inner race defect in a rolling element bearing," Journal of Tribology, vol. 138, no. 1, 2016.

[21] C. Mishra, A. K. Samantaray, and G. Chakraborty, "Ball bearing defect models: a study of simulated and experimental fault signatures," Journal of Sound and Vibration, vol. 400, pp. 86-112, 2017.

[22] Z. Luo, J. Guo, and R. Tang, "Research on nonlinear dynamic similarity of rolling ball bearings in rotor bearing system," Journal of Dynamics and Control, vol. 14, no. 3, pp. 223-228, 2016.

[23] Z. Guan, H. Zheng, and Y. Yang, "Bearing fault diagnosis based on empirical mode decomposition and duffing oscillator," Transactions of the Chinese Society for Agricultural Machinery, vol. 41, no. 9, pp. 214-217, 2010. 